\title{
Guidance for Industry \\ Considering Whether an FDA-Regulated Product Involves the Application of Nanotechnology
}

Contains Nonbinding Recommendations

June, 2014

Additional copies are available from:

Office of Policy

Office of the Commissioner

Food and Drug Administration

10903 New Hampshire Avenue

Silver Spring, MD 20993

Phone: 301-796-4830

http://www.fda.gov/RegulatoryInformation/Guidances/ucm257698.htm

You may submit electronic or written comments regarding this guidance at any time. Submit written comments on the guidance to the Division of Dockets Management (HFA-305), Food and Drug Administration, 5630 Fishers Lane, rm. 1061, Rockville, MD 20852. Submit electronic comments to http://www.regulations.gov. All comments should be identified with the docket number (FDA-2010-D-0530) listed in the notice of availability that publishes in the Federal Register.

For questions regarding this document contact: Office of the Commissioner, Food and Drug Administration, 10903 New Hampshire Avenue, Silver Spring, MD 20993, 301-796-4830.

\section{U.S. Department of Health and Human Services \\ Food and Drug Administration \\ Office of the Commissioner}

June 2014 


\section{TABLE OF CONTENTS}

\section{INTRODUCTION AND SCOPE}

II. DISCUSSION

\section{A. Points to Consider}

B. Rationale for Elements within the Points to Consider

1. Material or end product that is engineered to have certain dimensions or exhibit certain properties (in Points 1 and 2)

2. Material or end product (in Points 1 and 2)

3. At least one external dimension, or an internal or surface structure, in the nanoscale range (approximately $1 \mathrm{~nm}$ to $100 \mathrm{~nm}$ ) (in Point 1)

4. Properties or phenomena attributable to dimension(s) (in Point 2)

5. Dimension(s) of up to one micrometer $(1,000 \mathrm{~nm})$ (in Point 2)

\section{CONCLUSION \\ IV. REFERENCES}




\section{Guidance for Industry Considering Whether an FDA-Regulated Product Involves the Application of Nanotechnology ${ }^{1}$}

This guidance represents the Food and Drug Administration's (FDA's or the Agency's) current thinking on this topic. It does not create or confer any rights for or on any person and does not operate to bind FDA or the public. You can use an alternative approach if the approach satisfies the requirements of the applicable statutes and regulations. If you want to discuss an alternative approach, contact the FDA staff responsible for implementing this guidance. If you cannot identify the appropriate FDA staff, call the telephone number listed on the title page of this guidance.

\section{INTRODUCTION AND SCOPE}

Nanotechnology is an emerging technology that can be used in a broad array of FDA-regulated products, including medical products (e.g. to increase bioavailability of a drug), foods (e.g., to improve food packaging) and cosmetics (e.g. to affect the look and feel of cosmetics). Materials in the nanoscale range (i.e., with at least one dimension in the size range of approximately 1 nanometer (nm) to $100 \mathrm{~nm}$ ) can exhibit different chemical or physical properties, or biological effects compared to larger-scale counterparts. For example, dimension-dependent properties or phenomena may be used for functional effects such as increased bioavailability, decreased dosage, or increased potency of a drug product, decreased toxicity of a drug product, better detection of pathogens, more protective food packaging materials, or improved delivery of a functional ingredient or a nutrient in food (Refs. 1-6). These effects may derive from altered chemical, biological, or magnetic properties, altered electrical or optical activity, increased structural integrity, or other unique characteristics of materials in the nanoscale range not normally observed or expected in larger-scale materials with the same chemical composition (Ref. 7). Materials or end products may also exhibit similar properties or phenomena attributable to a dimension(s) outside the nanoscale range of approximately $1 \mathrm{~nm}$ to $100 \mathrm{~nm}$ (Refs. 27-30; see also discussion in Section II.B.5).

For the purpose of this guidance only, references to "products that involve the application of nanotechnology” or "nanotechnology products” mean products that contain or are manufactured using materials in the nanoscale range, as well as products that contain or are manufactured using certain materials that otherwise exhibit related dimension-dependent properties or phenomena. Likewise, we use the term "nanomaterial" generally to refer to both materials in the nanoscale

\footnotetext{
${ }^{1}$ This guidance finalizes the draft guidance, entitled "Draft Guidance for Industry: Considering Whether an FDARegulated Product Involves the Application of Nanotechnology,” which was issued in June, 2011. This guidance was prepared by FDA's Office of Policy in the Office of the Commissioner, in consultation with FDA's Center for Biologics Evaluation and Research, Center for Drugs Evaluation and Research, Center for Devices and Radiological Health, Center for Food Safety and Applied Nutrition, Center for Tobacco Products, Center for Veterinary Medicine, National Center for Toxicological Research, Office of the Chief Scientist, Office of Foods and Veterinary Medicine, Office of Regulatory Affairs, Office of Special Medical Programs, and Nanotechnology Task Force.
} 


\section{Contains Nonbinding Recommendations}

range and certain materials that otherwise exhibit related dimension-dependent properties or phenomena. Use of these terms is for the purpose of communicating FDA's current thinking elaborated in this document only.

As used in this guidance, the word "products" (or "FDA-regulated products") is meant to include products, materials, ingredients, and other substances regulated by FDA, including drugs, biological products, medical devices, food substances (including food for animals), dietary supplements, cosmetic products, and tobacco products. ${ }^{2}$

The guidance describes FDA's current thinking on determining whether FDA-regulated products involve the application of nanotechnology. This guidance is intended for manufacturers, suppliers, importers, and other stakeholders. (For convenience, the guidance will refer to these parties as "industry.”) FDA's guidance documents, including this guidance, do not establish legally enforceable responsibilities. Instead, guidance documents describe FDA's current thinking on a topic and should be viewed only as recommendations, unless specific regulatory or statutory requirements are cited. The use of the word should in Agency guidance documents means that something is suggested or recommended, but not required.

The application of nanotechnology may result in product attributes that differ from those of conventionally-manufactured products, and thus may merit particular examination. However, FDA (or "we") does not categorically judge all products that involve the application of nanotechnology as intrinsically benign or harmful. FDA will regulate nanotechnology products under existing statutory authorities, in accordance with the specific legal standards applicable to each type of product under its jurisdiction. We consider the current framework for safety assessment sufficiently robust and flexible to be appropriate for a variety of materials, including nanomaterials. FDA maintains a product-focused, science-based regulatory policy. Technical assessments will be product-specific, taking into account the effects of nanomaterials in the particular biological and mechanical context of each product and its intended use. As such, the particular policies for each product area, both substantive and procedural, will vary according to the statutory authorities and relevant regulatory frameworks (Ref. 8). We believe that this regulatory policy allows for tailored approaches that adhere to applicable legal frameworks and reflect the characteristics of specific products or product classes and evolving technology and scientific understanding.

This guidance provides an overarching framework for FDA's approach to the regulation of nanotechnology products. It identifies two points to consider when determining whether the FDA-regulated product involves the application of nanotechnology. An affirmative finding to either of the Points to Consider, elaborated in section II below, might suggest the need for particular attention by the Agency and/or industry to the product to identify and address potential implications for safety, effectiveness, public health impact, or regulatory status of the product.

This guidance does not address, or presuppose, what ultimate regulatory outcome, if any, will result in a particular case where the use of these points may indicate that an FDA-regulated product involves the application of nanotechnology. Issues such as the safety, effectiveness,

\footnotetext{
${ }^{2}$ Nanotechnology may also be applied to combination products (as defined at 21 CFR 3.2(e)).
} 


\section{Contains Nonbinding Recommendations}

public health impact, or the regulatory status of nanotechnology products are currently addressed on a case-by-case basis using FDA's existing review processes. ${ }^{3}$

This guidance also does not establish regulatory definitions. Rather, it is intended to help industry and others identify when they should consider potential implications for regulatory status, safety, effectiveness, or public health impact that may arise with the application of nanotechnology in FDA-regulated products. We advise industry to consult with FDA early in the development process to facilitate a mutual understanding of the specific scientific and regulatory issues for their nanotechnology products.

FDA will provide further guidance to industry, as needed, to address the application of nanotechnology as applicable to specific FDA-regulated products or classes of products (such as human foods, drugs, or cosmetics), consistent with existing federal policies (Refs. 9, 10). As appropriate, FDA's product-specific guidance documents will address issues such as the regulatory status, safety, effectiveness, performance, quality, or public health impact of nanotechnology products. ${ }^{4}$

\section{DISCUSSION}

FDA has not established regulatory definitions of "nanotechnology," “nanomaterial," "nanoscale," or other related terms. These terms are commonly used in relation to the engineering (i.e., deliberate manipulation, manufacture or selection) of materials that have at least one dimension in the size range of approximately 1 nanometers (nm) to $100 \mathrm{~nm}$. For example, the National Nanotechnology Initiative Program defines nanotechnology as "the understanding and control of matter at dimensions between approximately 1 and 100 nanometers, where unique phenomena enable novel applications” (Ref. 11). Various published definitions mention other factors such as function, shape, charge, the ratio of surface area to volume, or other physical or chemical properties.

Based on our current scientific and technical understanding of nanomaterials and their characteristics, FDA believes that evaluations of safety, effectiveness, public health impact, or regulatory status of nanotechnology products should consider any unique properties and behaviors that the application of nanotechnology may impart. This guidance identifies two Points to Consider that should be used to evaluate whether FDA-regulated products involve the application of nanotechnology. These points address both particle dimensions and dimensiondependent properties or phenomena. Product-specific premarket review, when required, offers an opportunity for FDA to apply these points and, where products are not subject to premarket review, industry should consider these points. If either point applies to a given product, industry

\footnotetext{
${ }^{3}$ It bears noting that the application of nanotechnology may also affect the classification of a product. For example, nanomaterials used in medical products may function through different modes of action than larger-scale materials with the same chemical composition, which may affect the classification of the product, for example as a drug or device.

${ }^{4}$ FDA's guidance documents relevant to nanotechnology, including product-specific guidance documents that focus on nanotechnology applications in specific product sectors, can be found at: http://www.fda.gov/ScienceResearch/SpecialTopics/Nanotechnology/default.htm
} 


\section{Contains Nonbinding Recommendations}

and FDA should consider whether the evaluations of safety, effectiveness, public health impact, or regulatory status of that product have identified and adequately addressed any unique properties or behaviors of the product.

These two Points to Consider are intended to provide an initial screening tool that can be broadly applied to all FDA-regulated products, with the understanding that these points are subject to change in the future as new information becomes available. In particular, FDA may further refine these points, either as applicable broadly to all FDA-regulated products or as applicable to particular products or classes of products, as justified by scientific information. This may include refining particle size parameters or introducing additional parameters such as those related to particle size distribution or specific properties. ${ }^{5}$ We will consider future revisions to our approach, including developing regulatory definitions relevant to nanotechnology, as warranted and in keeping with evolving scientific understanding. As previously indicated, FDA also may provide additional guidance, including product-specific guidance documents, to address issues such as the regulatory status, safety, effectiveness, performance, quality, or public health impact of nanotechnology products.

\section{A. Points to Consider}

At this time, when considering whether an FDA-regulated product involves the application of nanotechnology, FDA will ask:

1. Whether a material or end product is engineered to have at least one external dimension, or an internal or surface structure, in the nanoscale range (approximately $1 \mathrm{~nm}$ to $100 \mathrm{~nm}$ );

In addition, as we explain in more detail below, because materials or end products can also exhibit related properties or phenomena attributable to a dimension(s) outside the nanoscale range of approximately $1 \mathrm{~nm}$ to $100 \mathrm{~nm}$ that are relevant to evaluations of safety, effectiveness, performance, quality, public health impact, or regulatory status of products, we will also ask:

2. Whether a material or end product is engineered to exhibit properties or phenomena, including physical or chemical properties or biological effects, that are attributable to its dimension(s), even if these dimensions fall outside the nanoscale range, up to one micrometer $(1,000 \mathrm{~nm}){ }^{6}$

\footnotetext{
${ }^{5}$ At this time, we do not have an adequate basis on which to determine a particle number threshold or a list of "unique" or "novel" properties that are applicable across the range of FDA-regulated products. In addition, challenges related to measurement methods and biological effects add further complexity to recommending use of particle number, weight, or surface area as the most appropriate units of measure. FDA intends to actively follow scientific developments on this issue and provide additional guidance, as appropriate.

${ }^{6}$ As explained in section II.B.5. below, the use of $1,000 \mathrm{~nm}$ as a reference point should not be interpreted to mean that materials or products with dimensions above 1,000 nm cannot exhibit dimension-dependent properties or phenomena of importance to safety, effectiveness, public health impact, or regulatory status of the material or product. See further discussion on this issue in section II.B.5. below.
} 


\section{Contains Nonbinding Recommendations}

These considerations apply not only to new products, but also when changes to manufacturing processes alter the dimensions, properties, or effects of an FDA-regulated product or any of its constituent parts. ${ }^{7}$

\section{B. Rationale for Elements within the Points to Consider}

1. Material or end product that is engineered to have certain dimensions or exhibit certain properties (in Points 1 and 2)

The term "engineered," used in both Points 1 and 2, is used to distinguish products that have been deliberately manipulated by the application of nanotechnology from those products that contain materials that naturally occur in the nanoscale range. FDA is particularly interested in the deliberate and purposeful manipulation and control of dimensions to produce specific properties, because the emergence of these new properties or phenomena may raise questions about the safety, effectiveness, performance, quality or public health impact that may warrant further evaluation. FDA's interest in materials or products “engineered" to have nanoscale dimensions or related dimension-dependent properties or phenomena is distinct from the more familiar use of biological or chemical substances that may naturally exist at small scales, including at the nanoscale, such as microorganisms or proteins.

The term "engineered" is also used to distinguish products that have been deliberately manipulated by the application of nanotechnology from products that may unintentionally include materials in the nanoscale range.

For example, the incidental presence of particles in the nanoscale range in conventionallymanufactured products ${ }^{8}$ is not covered under the scope of this guidance. ${ }^{9}$

2. Material or end product (in Points 1 and 2)

The phrase "material or end product," referred to in both Points 1 and 2, is used to cover different types of articles that are regulated by FDA, such as products, materials, ingredients, and other substances regulated by FDA. This includes finished products (e.g., a drug tablet for administration to a patient) as well as materials that are intended for use in a finished product (e.g., a food additive added to a food during processing). In determining whether a material or end product satisfies either Point 1 or Point 2, FDA will examine the material or end product, and may also consider the constituent parts of the material or end product. Therefore, relevant considerations include whether a material or end product contains or involves in its manufacture the use of materials that meet either Point 1 or Point 2.

\footnotetext{
${ }^{7}$ These Points to Consider are not intended to apply to products that have been previously reviewed or approved by FDA and where no changes are made to manufacturing processes that would alter the dimensions, properties or effects of the product or its constituent parts.

${ }^{8}$ For example, small amounts of particles in the nanoscale range have been reported to be present in foods manufactured using conventional food manufacturing practices (Ref. 12).

${ }^{9}$ However, evaluations of conventionally-manufactured products may include a consideration of the effects, if any, of such incidental presence of particles in the nanoscale range on the safety, effectiveness, or public health impact of a product.
} 


\section{Contains Nonbinding Recommendations}

3. At least one external dimension, or an internal or surface structure, in the nanoscale range (approximately $1 \mathrm{~nm}$ to $100 \mathrm{~nm}$ ) (in Point 1)

A size range of approximately $1 \mathrm{~nm}$ to $100 \mathrm{~nm}$ is commonly used in various working definitions or descriptions regarding nanotechnology proposed by the regulatory and scientific community. ${ }^{10}$ In this size range, materials can exhibit new or altered physicochemical properties that can enable novel applications (Refs. 11, 13-15). Accordingly, per Point 1, if a material or end product is engineered to have at least one external dimension in the range of $1 \mathrm{~nm}$ to $100 \mathrm{~nm}$, or is engineered to have an internal or surface structure in the range of $1 \mathrm{~nm}$ to $100 \mathrm{~nm}$, industry and FDA should consider any unique characteristics or biological effects exhibited by the product that may influence its safety, effectiveness, public health impact, or regulatory status. Primary particles engineered with at least one external dimension within the nanoscale range are covered in Point 1 . This Point also covers any aggregates or agglomerates formed by such nanoscale primary particles. In addition, coated, functionalized, or hierarchically-assembled engineered structures that include internal or surface discrete and functional nanoscale entities, such as where such entities are embedded or attached to the surface, are encompassed within Point 1. ${ }^{11}$ Such engineered structures with discrete and functional nanoscale entities embedded or attached to the surface may have altered properties or phenomena that may affect product safety or effectiveness (Ref. 16). The inclusion of particles, objects, or structures with internal, surface, or external dimension(s) in the nanoscale range is consistent with approaches taken by other scientific and regulatory bodies (Refs. 17-23).

\section{4. $\quad$ Properties or phenomena attributable to dimension(s) (in Point 2)}

While size alone, for very small particles, is suggestive of the presence of properties meriting further examination, the identification and assessment of specific dimension-dependent properties and phenomena are ultimately more relevant for purposes of FDA regulatory review and oversight. Point 2, therefore, focuses on the properties of the material and its behavior in biological systems. ${ }^{12}$ The phrase "exhibits properties or phenomena . . . that are attributable to its dimension(s)," is used because properties and phenomena of materials in the nanoscale range enable applications that can affect the safety, effectiveness, performance, quality, public health impact, or regulatory status of FDA-regulated products. For example, as noted above, dimension-dependent properties or phenomena may be used for various functional effects such as increased bioavailability or decreased toxicity of drug products, better detection of pathogens,

\footnotetext{
${ }^{10}$ For example, a size range of approximately $1 \mathrm{~nm}$ to $100 \mathrm{~nm}$ is used in definitions, working definitions, or descriptions published by the National Nanotechnology Initiative (Ref. 11); Environmental Protection Agency (http://www.epa.gov/pesticides/regulating/nanotechnology.html); European Commission (Ref. 17); Health Canada (Ref. 19); International Standards Organization (Ref. 20); Organization for Economic Cooperation and Development's Working Party on Nanotechnology and Working Party on Manufactured Nanomaterials (http://www.oecd.org/sti/nano/); National Cancer Institute (http://www.cancer.gov/dictionary?cdrid=445071); and American National Standards Institute (http://nanostandards.ansi.org/tiki-index.php).

${ }^{11}$ This is not intended to include any incidental presence of internal or surface features with dimensions in the nanoscale range that may be present in conventionally-manufactured substances (for example, internal porosity, surface roughness or surface defects).

${ }^{12}$ Consistent with "Policy Principles for the U.S. Decision-Making Concerning Regulation and Oversight of Applications of Nanotechnology and Nanomaterials,” Office of Science and Technology Policy, Office of Management and Budget, and Office of the United States Trade Representative, June 9, 2011 (Ref. 10).
} 


\section{Contains Nonbinding Recommendations}

improved food packaging materials, or improved delivery of nutrients. These effects may derive from altered or unique characteristics of materials in the nanoscale range that are not normally observed or expected in larger-scale materials with the same chemical composition (Ref. 7). However, such changes may raise questions about the safety, effectiveness, performance, quality or public health impact of nanotechnology products. In addition, considerations such as routes of exposure, dosage, and behavior in various biological systems (including specific tissues and organs) (Refs. 13, 24) are critical for evaluating the safety, effectiveness, public health impact, or regulatory status of a wide array of products under FDA's jurisdiction. Such evaluations should include a consideration of the specific tests (whether traditional, modified, or new) that may be needed (Refs. 25, 26) to determine the physicochemical properties and biological effects of a product that involves the application of nanotechnology.

\section{Dimension(s) of up to one micrometer $(1,000 \mathrm{~nm})$ (in Point 2)}

Materials or end products can also exhibit properties or phenomena attributable to a dimension(s) outside the nanoscale range of approximately $1 \mathrm{~nm}$ to $100 \mathrm{~nm}$. Physical and chemical properties and biological behavior that are relevant to evaluations of safety, effectiveness, performance, quality, public health impact, or regulatory status of products have been observed at dimensions outside the nanoscale range of approximately $1 \mathrm{~nm}$ to $100 \mathrm{~nm}$ (Refs. 27-30). Therefore, Point 2 focuses on the importance of considering properties or phenomena attributable to dimensions, even where such dimensions may be outside the nanoscale range of approximately $1 \mathrm{~nm}$ to 100 nm. FDA's consideration of materials with dimension(s) outside the nanoscale range of approximately $1 \mathrm{~nm}$ to $100 \mathrm{~nm}$ is consistent with approaches taken by other scientific and regulatory organizations. ${ }^{13}$

\footnotetext{
${ }^{13}$ For example, the Joint Research Centre and the Scientific Committee on Emerging and Newly Identified Health Risks of the European Commission concluded: "In order to base a nanomaterials definition for regulatory purposes on size alone, the upper nanoscale limit should ideally be high enough to capture all types of materials that would need particular attention for regulation due to their nanoscale size. Upper limits which are often used in existing definitions, for example $100 \mathrm{~nm}$, may require the introduction of one or more qualifiers based on structural features or properties other than size, in order to capture structures of concern (for example agglomerates or aggregates) with a size larger than $100 \mathrm{~nm}$ in the regulation" (Ref. 22); “The upper size limit for one or more external dimensions of $100 \mathrm{~nm}$ is complicated by the potential exclusion of aggregates, agglomerates and multicomponent assemblies that would have external sizes greater than this" (Ref. 23); and “An upper limit of $100 \mathrm{~nm}$ is commonly used by general consensus but there is no scientific evidence to support the appropriateness of this value (Stated as SCENIHR conclusions in the European Commission Recommendation on the definition of nanomaterial, Ref. 17). The European Commission further noted that "it may be necessary to include additional materials, such as some materials with a size ... greater than $100 \mathrm{~nm}$ in the scope of application of specific legislation or legislative provisions suited for a nanomaterial (Ref. 17). In addition, the International Organization for Standardization (ISO) "acknowledged that health and safety considerations associated with intentionally produced and incidental nanoobjects do not abruptly end at dimensions of $100 \mathrm{~nm}$. As knowledge expands, it is abundantly clear that a robust terminology will need to capture and convey effectively the performance aspects of intentionally produced nanoobjects and nanostructured materials in their definitions, apart from their fundamental size and shape” (Ref. 20). More recently, Health Canada adopted a working definition of nanomaterial that, in part, reflects that it is possible for nanoscale properties/ phenomena to be exhibited outside the $1 \mathrm{~nm}$ to $100 \mathrm{~nm}$ size range, such as select quantum devices (Ref. 19). Finally, in its second regulatory review on nanomaterials, the European Commission noted that "fullerenes, graphene flakes and single wall carbon nanotubes with one or more external dimensions below $1 \mathrm{~nm}$ should be considered as nanomaterials.” Several types of nanomaterials were identified as not matching the EU definition, with an acknowledgment that "there are an increasing number of particles which are engineered to have internal nanoscale features. Examples are core-shell particles and nano-encapsulates. These particles may be designed, for example for pharmaceutical applications, where the inner core particle is "released" in a certain
} 


\section{Contains Nonbinding Recommendations}

At the present time, available scientific information does not establish a uniform upper boundary above $100 \mathrm{~nm}$ where novel properties and phenomena similar to those seen in materials with dimensions in the nanoscale range cease for all potential materials or end products. For this reason, at this time, FDA finds it reasonable to consider evaluation of materials or end products engineered to exhibit properties or phenomena attributable to dimensions up to $1,000 \mathrm{~nm}$, as a means to screen materials for further examination and to determine whether these materials exhibit properties or phenomena attributable to their dimension(s) and associated with the application of nanotechnology. ${ }^{14}$ An upper limit of one micrometer $(1,000 \mathrm{~nm})$ applied in the context of properties or phenomena attributable to dimensions serves both to: (1) include materials with dimension(s) outside the nanoscale range of approximately $1 \mathrm{~nm}$ to $100 \mathrm{~nm}$ that may exhibit dimension-dependent properties or phenomena associated with the application of nanotechnology and distinct from those of macro-scaled materials; and (2) exclude macro-scaled materials that may have properties attributable to their dimension(s) but are not likely associated with the application of nanotechnology.

An upper limit of 1,000 nm, combined with the presence of dimension-dependent properties or phenomena similar to those seen in materials with dimensions in the nanoscale range, provides an initial screening tool to help identify materials or products with properties or phenomena of particular relevance for regulatory review. The use of $1,000 \mathrm{~nm}$ as a reference point in this context should not be interpreted to mean that materials or products with dimensions above $1,000 \mathrm{~nm}$ cannot exhibit dimension-dependent properties or phenomena of importance to safety, effectiveness, public health impact, or regulatory status of the material or product. As noted above, we may further refine these Points to Consider, including this upper limit, either as applicable broadly to FDA-regulated products or as applicable to specific products or product categories.

\section{CONCLUSION}

The two Points to Consider elaborated in this guidance should be applied when considering whether an FDA-regulated product involves the application of nanotechnology. An affirmative finding to either of the Points to Consider, elaborated in this guidance, might suggest the need for particular attention to the product by FDA and/or industry for potential implications for safety, effectiveness, public health impact, or regulatory status of the product. We will consider future revisions to our approach, including developing regulatory definitions relevant to nanotechnology, as warranted and in keeping with evolving scientific understanding.

There remains a need to learn more about the potential role and importance of dimensions in the physical and chemical characteristics and biological effects exhibited by FDA-regulated products

environment. Some of these materials have an external diameter smaller than $100 \mathrm{~nm}$, matching the EU nanomaterial definition, others have an external diameter larger than $100 \mathrm{~nm}$, not matching the EU nanomaterial definition” (Ref. 31).

${ }^{14}$ However, as noted previously, FDA will consider further refinement of these Points to Consider for particular products or classes of products, as scientific information becomes available, including refining particle size parameters. 


\section{Contains Nonbinding Recommendations}

that involve the application of nanotechnology. ${ }^{15}$ Product-specific premarket review, when required, offers an opportunity for FDA to better understand the properties and behavior of products that involve the application of nanotechnology. Where products that involve the application of nanotechnology are not subject to premarket review, we urge industry to consult with the Agency early in the product development process. In this way, any questions about the products' regulatory status, safety, effectiveness, or public health impact can be appropriately and adequately addressed. FDA has and, as needed, will continue to provide additional guidance to industry in more targeted guidance documents to address these considerations.

\section{REFERENCES}

We have placed these references on display in the Division of Dockets Management, Food and Drug Administration, 5630 Fishers Lane, rm. 1061, Rockville, MD 20852. You may see them at that location between 9 a.m. and 4 p.m., Monday through Friday. As of June 1, 2014, FDA had verified the Web site addresses for the references it makes available as hyperlinks from the Internet copy of this guidance, but FDA is not responsible for any subsequent changes to NonFDA Web site references after June 1, 2014.

1. Merisko-Liversidge EM and Liversidge GG. Drug nanoparticles: formulating poorly water-soluble compounds. Toxicologic Pathology, 36:43-48, 2008.

2. Paciotti GF, Myer L, Weinreich D, et al. Colloidal gold: a novel nanoparticle vector for tumor directed drug delivery. Drug Delivery, 11:169-183, 2004.

3. Kaittanis C, Santra S, Manuel PJ. Emerging nanotechnology-based strategies for the identification of microbial pathogenesis. Advanced Drug Delivery Reviews 62:408-423, 2010.

4. Chaudhry Q, Scotter M, Blackburn J, et al. Applications and implications of nanotechnologies for the food sector. Food Additives and Contaminants 25:241-258, 2008.

5. Institute of Medicine. Nanotechnology in food products: Workshop Summary. Washington, DC: The National Academies Press, 2009.

6. Chen L, Remondetto GE, Subirade M. Food protein-based materials as nutraceutical delivery systems. Trends in Food Science \& Technology 17:272-283, 2006.

7. Nanotechnology. A Report of the U.S. Food and Drug Administration Nanotechnology Task Force, July 25, 2007; available online at: http://www.fda.gov/ScienceResearch/SpecialTopics/Nanotechnology/UCM2006659.htm

\footnotetext{
${ }^{15}$ FDA's nanotechnology regulatory science program aims to further enhance FDA's scientific capabilities, including developing necessary data and tools to identify and measure dimension-dependent properties and assess their potential impact on safety or effectiveness. See http://www.fda.gov/ScienceResearch/SpecialTopics/Nanotechnology/ucm273325.htm
} 


\section{Contains Nonbinding Recommendations}

8. FDA's Approach to Regulation of Nanotechnology Products; available online at: http://www.fda.gov/ScienceResearch/SpecialTopics/Nanotechnology/ucm301114.htm

9. Office of Science and Technology Policy, Office of Management and Budget, and the United States Trade Representative. Principles for Regulation and Oversight of Emerging Technologies, March 11, 2011; available online at: http://www.whitehouse.gov/sites/default/files/omb/inforeg/for-agencies/Principles-for$\underline{\text { Regulation-and-Oversight-of-Emerging-Technologies-new.pdf }}$

10. Office of Science and Technology Policy, Office of Management and Budget, and the United States Trade Representative. Policy Principles for the U.S. Decision-Making Concerning Regulation and Oversight of Applications of Nanotechnology and Nanomaterials, June 9, 2011; available online at: http://www.whitehouse.gov/sites/default/files/omb/inforeg/for-agencies/nanotechnologyregulation-and-oversight-principles.pdf

11. National Nanotechnology Initiative Strategic Plan, February 2014; available online at http://nano.gov/sites/default/files/pub_resource/2014_nni_strategic_plan.pdf

12. Palashuddin M, Jaiswal A, Paul A, et al. Presence of amorphous carbon nanoparticles in food caramels. Scientific Reports 2(383):1-5, 2012.

13. Kunzman A, Andersson B, Thurnherr T, et al. Toxicology of engineered nanomaterials: Focus on biocompatibility, biodistribution and biodegradation. Biochimica et Biophysica Acta 1810:361-373, 2011.

14. Myers JD, Doane T, Burda C, and Basilion JP. Nanoparticles for imaging and treating brain cancer. Nanomedicine 8(1):123-143, 2013.

15. Powers KW, Brown SC, Krishna VB, et al. Research Strategies for Safety Evaluation of Nanomaterials. Part VI. Characterization of Nanoscale Particles for Toxicological Evaluation. Toxicological Sciences 90: 296-303, 2006.

16. Small is Different: A Science Perspective on the Regulatory Challenges of the Nanoscale. Report of the Expert Panel on Nanotechnology. The Council of Canadian Academies, September, 2008; available online at: http://www.scienceadvice.ca/en/assessments/completed/nanotechnology.aspx.

17. European Commission Recommendation on the definition of nanomaterial, October, 2011; available online at: http://ec.europa.eu/environment/chemicals/nanotech/pdf/commission_recommendation.p $\underline{\mathrm{df}}$

18. Communication from the Commission to the European Parliament, the Council and the European Economic and Social Committee, Second Regulatory Review on 


\section{Contains Nonbinding Recommendations}

Nanomaterials, October 2012; available online at:

http://ec.europa.eu/nanotechnology/pdf/second_regulatory_review_on_nanomaterials_com(2012)_572.pdf

19. Policy Statement on Health Canada’s Working Definition for Nanomaterial, 2011; available online at: http://www.hc-sc.gc.ca/sr-sr/pubs/nano/pol-eng.php

20. International Organization for Standardization/Technical Specification, Nanotechnologies-Vocabulary-Part 1: Core terms, 2010 (ISO/TS 80004-1:2010).

21. Australia's National Industrial Chemicals Notification and Assessment Scheme's working definition of industrial nanomaterial, 2010; available online at: http://nanotech.lawbc.com/2010/10/articles/international/other/australia-announcesadjustments-to-nicnas-new-chemicals-processes-for-industrial-nanomaterials/

22. Considerations on a Definition of Nanomaterial for Regulatory Purposes, Joint Research Centre, 2010; available online at: http://ec.europa.eu/dgs/jrc/downloads/jrc_reference_report_201007_nanomaterials.pdf

23. Scientific Basis for the Definition of the Term "Nanomaterial”, Scientific Committee on Emerging and Newly Identified Health Risks, 2010; available online at: http://ec.europa.eu/health/scientific_committees/emerging/docs/scenihr_o_030.pdf

24. Nel AE, Madler L, Velegol D, et al. Understanding biophysicochemical interactions at the nano-bio interface. Nature Materials 8:543-557, 2009.

25. Khushf G and Siegel RA. What is unique about nanomedicine? The significance of the mesoscale. Journal of Law, Medicine, \& Ethics 40(4)780-794, 2012.

26. Mauer-Jones MA and Haynes CL. Toward correlation in in vivo and in vitro nanotoxicology studies. Journal of Law, Medicine, \& Ethics 40(4)795-801, 2012.

27. Etheridge ML, Campbell SA, Erdman AG, et al. The big picture on nanomedicine: the state of investigational and approved nanomedicine products. Nanomedicine: Nanotechnology, Biology, and Medicine 9:1-14, 2013.

28. Almeida JPM, Chen AL, Foster A, and Drezek R. In vivo biodistribution of nanoparticles. Nanomedicine 6(5):815-835, 2011.

29. Sonavane G, Tomoda K, and Makino K. Biodistribution of colloidal gold nanoparticles after intravenous administration: effect of particle size. Colloids and Surfaces B: Biointerfaces 66:274-280, 2008.

30. Liu Z, Chen K, Davis C, et al. Drug delivery with carbon nanotubes for in vivo cancer treatment. Cancer Research 68(16):6652-6660, 2008. 


\section{Contains Nonbinding Recommendations}

31. European Commission Staff Working Paper, Types and uses of nanomaterials, including safety aspects, October, 2012; available online at:

http://ec.europa.eu/nanotechnology/pdf/second_regulatory_review_on_nanomaterials__staff_working_paper_accompanying_com(2012)_572.pdf 